\title{
Fractional branes, warped compactifications and backreacted orientifold planes
}

\author{
J. Blåbäck, ${ }^{a}$ B. Janssen, ${ }^{b}$ T. Van Riet ${ }^{c}$ and B. Vercnocke ${ }^{c}$ \\ ${ }^{a}$ Institutionen för fysik och astronomi, Uppsala Universitet, \\ Box 803, SE-751 08 Uppsala, Sweden \\ ${ }^{b}$ Departamento de Física Teórica y del Cosmos and \\ Centro Andaluz de Física de Partículas Elementales, Universidad de Granada, 18071 Granada, \\ Spain \\ ${ }^{c}$ Institut de Physique Théorique, CEA Saclay, \\ CNRS URA 2306 \\ F-91191 Gif-sur-Yvette, France \\ E-mail: johan.blaback@physics.uu.se, bjanssen@ugr.es, \\ thomas.van-riet@cea.fr, bert.vercnocke@cea.fr
}

ABSTRACT: The standard extremal $p$-brane solutions in supergravity are known to allow for a generalisation which consists of adding a linear dependence on the worldvolume coordinates to the usual harmonic function. In this note we demonstrate that remarkably this generalisation goes through in exactly the same way for $p$-branes with fluxes added to it that correspond to fractional $p$-branes. We relate this to warped orientifold compactifications by trading the $\mathrm{D} p$-branes for $\mathrm{O} p$-planes that solve the $\mathrm{RR}$ tadpole condition. This allows us to interpret the worldvolume dependence as due to lower-dimensional scalars that flow along the massless directions in the no-scale potential. Depending on the details of the fluxes these flows can be supersymmetric domain wall flows. Our solutions provide explicit examples of backreacted orientifold planes in compactifications with non-constant moduli.

KEYwords: Flux compactifications, Supergravity Models, Superstring Vacua

ArXiv EPRINT: 1207.0814 


\section{Contents}

1 Introduction $\quad 1$

2 Warm up: dynamical branes 2

3 Generalised fractional brane solutions 3

4 Relation with warped compactifications $\quad 6$

4.1 General idea 6

$\begin{array}{ll}4.2 & \text { Explicit example }\end{array}$

$\begin{array}{lll}\text { 4.2.1 Three-scalar truncation } & 7\end{array}$

$\begin{array}{lll}4.2 .2 & \text { Some special domain wall flows } & 9\end{array}$

$\begin{array}{ll}4.2 .3 \text { Supersymmetry } & 10\end{array}$

$\begin{array}{lll}4.3 & \text { Interpretation } & 10\end{array}$

5 Outlook 12

$\begin{array}{ll}\text { A Ricci tensor } & 14\end{array}$

\section{Introduction}

Many semi-realistic compactifications of string theory involve brane or orientifold sources. Such compactifications are necessarily warped compactifications as the backreaction of the sources generates (amongst other things) the warp factor. Warping, if strong enough, can resolve mass hierarchy problems [1] or scale down susy breaking ingredients [2] in order to gain control over corrections to the effective action. Unfortunately the presence of the warp factor and other backreaction effects implies we cannot carry out the usual Kaluza-Klein dimensional reduction to construct the low energy effective theory, which is also named the warped effective field theory (WEFT). The current understanding of warped effective field theory is incomplete and the state of the art can be found in the references $[3 ?-18]{ }^{1}$ What is especially worrysome is that one expects warping corrections to be more relevant when supersymmetry is broken, such as in the KKLT proposal for dS vacua [2]. One could speculate that the warping corrections to the cosmological constant are proportional to the susy breaking scale and hence the corrections could be equal size as the bare cosmological constant. The reason warping corrections could be worse for non-supersymmetric solutions, and especially dS vacua, are based on experience with existing solutions [20] and on a simple 10-dimensional argument for classical dS solutions [21]. What has been pointed out in [20] is that for many known compactifications that are supersymmetric the unwarped limit (in

\footnotetext{
${ }^{1}$ A nice illustration for our incomplete understanding of warped effective field theory can be found in [18].
} 
which sources are smeared) at least captures the correct on-shell value for the moduli and the cosmological constant. WEFT instead becomes important when one tries to understand fluctuations around these supersymmetric (or fake supersymmetric) vacua, for example when computing moduli masses. This implies that the warped effective scalar potential coincides with the unwarped scalar potential only at those special BPS points in moduli space. We refer to [8] for an explicit example (see figure 1 in section 4.3 of that paper.)

One method to develop or test WEFT proposals is to construct fully backreacted tendimensional solutions and then dimensionally reduce them to check the consistency of the lower-dimensional WEFT. This strategy has been followed in $[5,6,15,18,20,22]$. It is the aim of this paper to continue with this strategy and take a first step towards constructing general non-trivial backreacted solutions that do not describe critical points of the lowerdimensional theory but dynamical solutions with non-constant scalars (for instance domain walls and cosmologies). In this paper we focus on the solutions and their interpretation in the WEFT, but the actual application to constructing and testing WEFT will be done elsewhere.

The rest of the paper is organised as follows. In section 2 we summarise the so called $d y$ namical $p$-brane solutions $[5,6,18,22,23]$. These are solutions where, in comparison to the standard $p$-brane solutions, a linear, worldvolume dependent function $H^{W}$ has been added to the harmonic function $H$. We then extend these solutions in section 3 to also include fluxes, also known as fractional branes. Solutions of this type are known ( $p=3$ in [22], and $p=6$ in [24]), but we generalise the solutions to also include $p=1,2,4,5$ in a single framework. We present the general solution and give some explicit examples for general $p$. The paper then continues in section 4 with a discussion on how these solutions relate to warped compactifications. The idea is that once fluxes are added one can trade the $\mathrm{D} p$-branes for $\mathrm{O} p$-planes and make the internal space compact. We use this to construct lower-dimensional effective theories in $p+1$ dimensions, in the smeared limit. We show that the worldvolume dependent function $H^{W}$ will imply a running of the scalars of the effective theory, and also discuss how $H^{W}$ is sometimes necessary to assure supersymmetry. The results and possible implications are then summarised in section 5 . For the reader interested in verifying the solutions of sections 2 and 3, we present the expression for the Ricci tensor in the appendix.

\section{Warm up: dynamical branes}

In this section we recall the standard extremal $p$-brane solutions in IIA and IIB for $p=$ $0, \ldots, 6$ and an extension thereof constructed in $[5,6,18,22,23]$, which has been named "dynamical $p$-branes" by some authors. We present the solutions as magnetic solitons in Einstein frame. The coordinates along the worldvolume are denoted $x^{a}$ with $a=1, \ldots, p+1$ and the coordinates along the transversal space are $y^{i}$ with $i=1, \ldots, 9-p$. The solutions are then given by the following expressions: ${ }^{2}$

$$
\begin{aligned}
\mathrm{d} s_{10}^{2} & =H^{\frac{p-7}{8}} \mathrm{~d} s_{p+1}^{2}+H^{\frac{p+1}{8}} \mathrm{~d} s_{9-p}^{2}, \\
\mathrm{e}^{\phi} & =g_{s} H^{\frac{3-p}{4}} \\
F_{8-p} & =-g_{s}^{\frac{3-p}{4}} \star_{9-p}\left(\partial_{i} H \mathrm{~d} y^{i}\right) .
\end{aligned}
$$

\footnotetext{
${ }^{2}$ We use the conventions and equations of motion as presented in [20].
} 
Where $\star_{9-p}$ is with respect to the transversal space metric $\mathrm{d} s_{9-p}^{2} \cdot{ }^{3}$ Both the worldvolume metric and transversal metric are taken to be Ricci flat:

$$
\begin{aligned}
\mathrm{d} s_{p+1}^{2} & =g_{a b}^{W} \mathrm{~d} x^{a} \mathrm{~d} x^{b}, & R_{a b}\left(g^{W}\right) & =0, \\
\mathrm{~d} s_{9-p}^{2} & =g_{i j}^{T} \mathrm{~d} y^{i} \mathrm{~d} y^{j}, & R_{i j}\left(g^{T}\right) & =0 .
\end{aligned}
$$

The function $H$ is, by the off-diagonal components of Einsteins equations, forced to have the form of a sum

$$
H(x, y)=H^{W}(x)+H^{T}(y),
$$

where $H^{T}$ is a general harmonic on the transversal space

$$
\nabla_{i} \partial^{i} H^{T}=0
$$

and $H^{W}(x)$ is a worldvolume-dependent harmonic function of a very special kind [23]

$$
\nabla_{a} \partial_{b} H^{W}=0
$$

The most well known solutions are those corresponding to string theory $\mathrm{D} p$-branes in flat space, for $p=0, \ldots, 6$. Then $g^{W}$ is the Minkowski metric, $g^{T}$ the metric on flat Euclidean space and the harmonic functions are

$$
H^{W}=0, \quad H^{T}(r)=1+\frac{Q}{r^{7-p}},
$$

where $r$ is the radial coordinate in the transversal space.

For a flat worldvolume the solution for $H^{W}$ is most easily written in Cartesian coordinates

$$
H^{W}=\sum_{a} c_{a} x^{a}
$$

where $c_{a}$ are constants. Since $x^{0}$ is the time-direction we can for instance make timedependent brane solutions in this way [25].

\section{Generalised fractional brane solutions}

We extend the above known dynamical $p$-brane solutions to solutions with extra fluxes. These extra fluxes can be interpreted in some cases as $(p+2)$-branes wrapping (collapsing) 2 -cycles in the transversal space. Such solutions are also known as fractional branes, famous examples include fractional D3 branes [27? ] and fractional M2 branes [28]; see [29] for a discussion of fractional brane solutions of various dimensionality. In this section we construct such solutions with non-zero $H^{W}$. To our knowledge most of these solutions are new. The $p=6$ solution with a specific choice of $H^{W}$ has been constructed before [24] and the $p=3$ solutions have appeared in [22].

\footnotetext{
${ }^{3}$ For $p=3$ it is understood that we have to add the self-dual piece to the $F_{5}$ expression in order to make $F_{5}$ self-dual $\left(F_{5}=\star_{10} F_{5}\right)$. From here on we suppress from writing this extra term for $p=3$ explicitly.
} 
One way to understand the existence of fractional brane solutions is by properly interpreting the Bianchi identity for a $F_{8-p}$ RR field strength:

$$
\mathrm{d} F_{8-p}=H_{3} \wedge F_{6-p}+\text { delta-source terms . }
$$

We notice that a suitable combination of $F_{6-p}$ and $H_{3}$ flux acts as a regularised magnetic source for $F_{8-p}$, in exactly the same way as a $\mathrm{D} p$-brane source smeared over all transversal directions. This suggest that a suitable flux choice might be mutually BPS with D $p$-brane sources and could therefore be added to the usual $\mathrm{D} p$-solution. This is an essential ingredient in for instance the Klebanov-Strassler background [27] and all its related solutions, where a suitable combination of $F_{3}$ and $H_{3}$ is added to D3 brane backgrounds.

The suitable choice of fluxes that is mutually BPS with $\mathrm{D} p / \mathrm{O} p$ sources is such that [20]

$$
F_{6-p}=g_{s}^{-\frac{p+1}{4}} \star_{9-p} H_{3} .
$$

This is the generalization of the imaginary self-duality (ISD) condition for the complex three-form for $p=3$. For the other fields in the solution we again take the Ansatz (2.1)-(2.3).

In summary we have ${ }^{4}$

$$
\begin{aligned}
\mathrm{d} s_{10}^{2} & =H^{\frac{p-7}{8}} \mathrm{~d} s_{p+1}^{2}\left(x^{a}\right)+H^{\frac{p+1}{8}} \mathrm{~d} s_{9-p}^{2}\left(y^{i}\right), \\
\mathrm{e}^{\phi} & =g_{s} H^{\frac{3-p}{4}}, \\
F_{8-p} & =-g_{s}^{\frac{3-p}{4}} \star_{9-p}\left(\partial_{i} H \mathrm{~d} y^{i}\right), \\
F_{6-p} & =g_{s}^{-\frac{p+1}{4}} \star_{9-p} H_{3},
\end{aligned}
$$

where again the transversal and worldvolume metrics are Ricci flat and the function $H$ is given by a sum $H=H^{W}(x)+H^{T}(y)$. We still have that $H^{W}$ satisfies (2.8):

$$
H^{W}=\nabla_{a} \partial_{b} H^{W}=0
$$

but the equation for $H^{T}$ is altered as follows:

$$
\left[\nabla^{T}\right]^{i} \partial_{i} H^{T}=-\frac{1}{3 !} g_{s}^{-1}\left|H_{3}\right|_{T}^{2},
$$

up to the source term. The contraction in $\left|H_{3}\right|_{T}^{2}$ is done using the transversal metric $g^{T}$.

The above Ansatz does not yet solve all equations of motion: the Einstein equations put non-trivial conditions on the transversal space. It must have the proper cycles to support the fluxes $F_{6-p}$ and $H$. Let us construct some explicit simple examples to see how that goes. As an internal space we choose a Ricci flat cone over a direct product of Einstein spaces:

$$
\mathrm{d} s_{9-p}^{2}=\mathrm{d} r^{2}+r^{2} g_{I J}^{(2)} \mathrm{d} y^{I} \mathrm{~d} y^{J}+r^{2} g_{\alpha \beta}^{(6-p)} \mathrm{d} y^{\alpha} \mathrm{d} y^{\beta} .
$$

${ }^{4}$ A notational subtlety arises when $p=2$. Then $F_{4}$ and $F_{6}$ should not be seen as separate forms. To write the solution more correct one could for instance just use $F_{4}$ and add the Hodge dual expression for $F_{6}$ to it. 
For this to be Ricci flat we must have the following curvatures on the separate Einstein spaces

$$
R_{I J}^{(2)}=(8-p) g_{I J}^{(2)}, \quad R_{\alpha \beta}^{(6-p)}=(8-p) g_{\alpha \beta}^{(6-p)} .
$$

One can for instance use $n$-spheres with proper curvature radii. Note that for $p=5$ this procedure does not work since there will be a one-dimensional subspace which cannot have non-zero curvature. For $p=6$ we just have one Einstein space and, if it is taken to be the unit 2-sphere, the transverse space is flat $\mathbb{R}^{3}$.

We then find that the equations of motion are solved by the natural choice of fluxes

$$
F_{6-p}=m \epsilon_{6-p}, \quad H_{3}=(-1)^{p} g_{s}^{\frac{p+1}{4}} m r^{p-4} \mathrm{~d} r \wedge \epsilon_{2},
$$

with $m$ a quantised flux number and $\epsilon_{6-p}$ and $\epsilon_{2}$ are volume forms on the two Einstein spaces. For $p=1,2,4,6$, we have explicitly

$$
H^{T}=C_{2}+\frac{Q}{r^{7-p}}-\frac{g_{s}^{\frac{p-1}{2}} m^{2}}{2(p-3)(p-5)} \frac{1}{r^{10-2 p}},
$$

where $C_{2}$ in arbitrary and $Q$ determines the charge of the $p$-brane. For asymptotically flat backgrounds we can set $C_{2}=1$. Note that for $p=3$ the last term becomes a logarithm:

$$
H^{T}=C_{2}+\frac{Q}{r^{4}}+\frac{m^{2}}{16 r^{4}}(4 \ln r+1) .
$$

The above solutions with $H^{W}=0$ described by equations (3.12), (3.13) were discussed before by Herzog and Klebanov in [29]. Note that supersymmetry requires the choice of a suitable conical internal space. Our simple explicit choice for the transverse space, a cone over a direct product of Einstein spaces (3.9), breaks all supersymmetry. However, by taking appropriate internal spaces, it should be possible to construct supersymmetric solutions with worldvolume dependence $H^{W}$. We leave the details to future work. For instance for $p=3$, if we choose the internal space to be a cone over $T^{1,1}$, there should be a supersymmetric extension of the Klebanov-Tseytlin and its Klebanov-Strassler resolution with a linear spatial worldvolume dependence.

There is however an exception for the solution with $p=6$. For $p=6$, reference [29] claims that there is no solution although it was known before by Janssen, Meessen and Ortín (JMO) in a slightly generalised form [24]. The solution in [24] was characterised by the following choice for $H$

$$
H(r, z)=1+\frac{Q}{r}-\frac{1}{6} m^{2} r^{2}+c z,
$$

where $z$ is a Cartesian spatial coordinate on the D6 worldvolume, $m$ is the Romans mass and $c$ a constant. We notice that this is exactly an example of adding a linear worldvolume dependence to the fractional brane solution. When $m$ and $c$ both vanish this is the standard extremal D6 solution in IIA supergravity, which preserves $1 / 2$ of the supersymmetry. When $m \neq 0$ it was shown that maximally $1 / 4$ of the supersymmetry could be preserved if

$$
c=m \text {. }
$$


Below we give an interpretation to this worldvolume dependence in terms of lower dimensional solutions to warped compactifications. This also allows us to understand when the worldvolume dependence is required for supersymmetry.

\section{Relation with warped compactifications}

\subsection{General idea}

Ten-dimensional metrics of the kind (2.1) can be regarded as warped compactifications down to $p+1$ dimensions if the transversal metric, including its conformal factor, can be interpreted as a metric on a compact space $\mathcal{M}_{9-p}$. For such compact solutions the integrated Bianchi identity implies a non-trivial global constraint (Gauss' law):

$$
\int_{\mathcal{M}_{9-p}} H_{3} \wedge F_{6-p}+Q_{\text {total }}=0
$$

where $Q_{\text {total }}$ is the integral over all delta-sources.

This is the so-called tadpole condition. For fluxes satisfying the BPS condition (3.2) this cannot be satisfied for $\mathrm{D} p$-brane sources. If one instead uses $\mathrm{O} p$-plane sources one can satisfy the tadpole condition and obtain a stable compactification. For $p=3$ this is the well-known GKP compactification [1] and the $p=1,2,4,5,6$ solutions are formally related by T-duality and described in [20].

If one considers these solutions instead in a non-compact setting and takes them to be spherically symmetric, then these are given by the brane solutions of the previous section, but with $Q<0$. This implies that these solutions have unphysical regions, since for small enough $r$ :

$$
H^{T}(r) \rightarrow 1-\frac{|Q|}{r^{7-p}}<0 .
$$

In some occasions one can hope that a lift to M-theory resolves this unphysical singularity or that the non-zero fluxes in the background resolve these singularities as in [30]. For the solutions in this paper this does not seem to be the case.

Effectively one can think of a map between two sets of BPS solutions that one obtains by trading $\mathrm{D} p$ sources for $\mathrm{O} p$ sources. In practice this means flipping the sign of the $|Q| r^{p-7}$ term in the transversal harmonic function $H^{T}$. For both $\mathrm{O} p$ and $\mathrm{D} p$ sources, the fluxes (3.2) are mutually BPS with the source. For D $p$-branes the fluxes and the source gravitationally attract but electromagnetic forces counter balance this attraction. For $\mathrm{O} p$-planes it is the other way around. In what follows we perform this flip and look at the fractional brane solutions in which the $\mathrm{D} p$ source has been turned into an $\mathrm{O} p$ source and the transversal space is taken to be compact. This allows us to define an effective field theory on the $(p+1)$-dimensional worldvolume. Due to the presence of the fluxes these are theories with non-zero scalar potentials for the moduli.

Clearly, when we consider solutions with $H^{W}=0$ we just have a compactification down to $(p+1)$-dimensional Minkowski space. These are the so-called no-scale Minkowski solutions. What we are after is the interpretation of solutions with $H^{W} \neq 0$. It is tempting to interpret the $x^{a}$ dependence as the dependence of some lower-dimensional scalars on the 
coordinates $x^{a}$. Hence, instead of being stuck at the Minkowski minimum, the scalars are non-constant. Schematically we have:

$$
\begin{aligned}
& H^{W}=0 \quad \Longleftrightarrow \quad \text { No-scale Minkowski solution, } \\
& H^{W} \neq 0 \quad \Longleftrightarrow \quad \text { Non-constant scalar fields. }
\end{aligned}
$$

We demonstrate this with an explicit example below. We do not expect the general solution with running scalars to lift to the 10D solution with non-zero metric. Only solutions with specific moduli turned on will fit into this class as we explain below.

This interpretation shows that one has to be careful with interpreting what is the lower-dimensional metric in (2.1). When there is $x^{a}$-dependence then $\mathrm{d} s_{p+1}^{2}\left(x^{a}\right)$ is the Minkowski metric in our solutions. However, it should not be interpreted as the lowerdimensional metric because the volume modulus is $x^{a}$-dependent and one needs to correct for this if one goes to the Einstein frame metric. Therefore the lower-dimensional metric is conformal Minkowski, with a conformal factor related to the volume modulus. This makes sense since a lower-dimensional Minkowski metric is inconsistent with the assumption of flowing scalar fields.

Special choices of $H^{W}$ lead to different lower-dimensional solutions. For example, when $H^{W}$ is linear in time, this corresponds to a specific FLRW solution. When it is linear in a spatial coordinate instead it describes a domain wall like solution

$$
\begin{array}{lll}
H^{W} \propto t & \Longleftrightarrow & \text { FLRW compactification, } \\
H^{W} \propto z & \Longleftrightarrow & \text { domain wall compactification. }
\end{array}
$$

When both $t$ and $z$ dependence is present the solution is more difficult to interpret.

\subsection{Explicit example}

To illustrate the above we consider a very simple truncation of the effective theory down to three scalars. We do this by taking simple fluxes consistent with the BPS relation (3.2). We furthermore perform the dimensional reduction assuming the orientifold is smeared and therefore ignore all issues related to warped effective field theory. The reason is that the fully localised (warped) solutions are presented above and hence known. What we want to demonstrate is that the solutions of the lower-dimensional smeared compactification give exactly the $x^{a}$-dependence of the $H^{W}$ function in the 10-dimensional solution. The effect of the localisation (and hence full backreaction) of the $\mathrm{O} p$-plane is simply to add the $H^{T}$ piece to the solution. See [20] for an extensive discussion on smeared versus localised orientifold solutions. The dynamical fractional brane solutions provide new examples in which the localised versus smeared source limit is understood from a 10-dimensional point of view. We plan to elaborate on this in future work.

\subsubsection{Three-scalar truncation}

There exist three obvious moduli: the dilaton $\phi$, the volume modulus of the internal $(9-p)$ dimensional space, called $v$ and the volume of the cycle wrapped by the $F_{6-p}$-flux, called 
$\chi$. The metric Ansatz, in 10-dimensional Einstein frame, is given by

$$
\mathrm{d} s_{10}^{2}=\exp (2 \alpha v) \mathrm{d} s_{p+1}^{2}+\exp (2 \beta v)\left\{\exp (\gamma \chi) \mathrm{d} s_{3}^{2}+\exp (\delta \chi) \mathrm{d} s_{6-p}^{2}\right\},
$$

with the numbers $\alpha, \beta, \gamma, \delta$ chosen such that we end up in lower-dimensional Einstein frame with canonically normalised fields:

$$
\beta=-\frac{(p-1)}{9-p} \alpha, \quad \alpha^{2}=\frac{9-p}{16(p-1)}, \quad \gamma=-\frac{\delta(6-p)}{3}, \quad \delta^{2}=\frac{6}{(9-p)(6-p)} .
$$

We have made a very strong simplifying assumption: the $H_{3}$ field strength fills the 3dimensional subspace with metric $\mathrm{d} s_{3}^{2}$ and the $F_{6-p}$ flux fills the subspace with metric $\mathrm{d} s_{6-p}^{2}$. This is a very simple flux Ansatz, that allows us to find a consistent truncation down to the three moduli $\phi, v, \chi$. This consistent truncation is only possible in the smeared limit. In the warped case there might not exist a simple lower-dimensional truncation and even the degrees of freedom are not clear [18]. Hence it remains to be seen whether any reliable information can be obtained from this truncation. As we check below, this truncation is capable of describing the linear dependence in $H^{W}$, but not more.

The lower-dimensional action is obtained from a direct dimensional reduction of the type II supergravity action with a smeared $\mathrm{O} p$ source

$$
S=\int \sqrt{-g}\left(R-\frac{1}{2}(\partial \phi)^{2}-\frac{1}{2}(\partial \chi)^{2}-\frac{1}{2}(\partial v)^{2}-V(\phi, v, \chi)\right) .
$$

The scalar potential $V$ gets contributions from the fluxes and the negative orientifold plane tension and has the form of an exact square (due to the tadpole condition). To write it down in a clean way we perform the following $\mathrm{SO}(3)$ field rotation $(\phi, v, \chi) \rightarrow(x, u, w)$ :

$$
\begin{aligned}
& \phi=-\frac{(p-3)}{4} \sqrt{\frac{p-1}{2 p}} x+\frac{p+1}{8} u+\frac{p+1}{8} \sqrt{\frac{3(6-p)}{p}} w, \\
& v=-(p+1) \sqrt{\frac{9-p}{32 p}} x-\frac{p-3}{8} \sqrt{\frac{p-1}{9-p}} u-\frac{p-3}{8} \sqrt{\frac{3(6-p)(p-1)}{(9-p) p}} w, \\
& \chi=-\frac{1}{2} \sqrt{\frac{6(6-p)}{9-p}} u+\sqrt{\frac{p}{2(9-p)}} w .
\end{aligned}
$$

The scalar potential then becomes

$$
V(x, u, w)=\frac{1}{2} \exp \left(-2 \sqrt{\frac{p}{2(p-1)}} x\right)[\mathcal{H} \exp (-u)-\mathcal{F} \exp (+u)]^{2},
$$

where $\mathcal{H}$ and $\mathcal{F}$ represent the flux densities and are positive numbers. We furthermore observe that the scalar potential only depends on two scalars $(u$ and $x)$ instead of three. The scalar potential (4.11) is of the no-scale type and at the no-scale Minkowski vacuum we have $\exp (2 u)=\frac{\mathcal{H}}{\mathcal{F}}$, whereas $x$ and $w$ have arbitrary constant values.

This lower-dimensional action is expected to be a truncation of a half maximal gauged supergravity in $p+1$ dimensions for which the scalar coset is truncated to three scalar fields 
spanning three flat directions. For the case $p=3$ this half maximal gauged supergravity has been worked out in all detail in [31].

For the special case $p=6$, which is an O6 compactification to 7 dimensions, the $\chi$ modulus is absent and we have a two-scalar truncation instead. From here on we keep the notation general and include $\chi$ with the understanding that it is absent when $p=6$. Our flux Ansatz is the most general one for $p=6$, but for $p<6$ more general flux choices exist. This will be relevant later when we discuss the supersymmetry of the solutions. It turns out that the current flux choices do not allow for supersymmetric Minkowski solutions. However, they allow for domain wall flows that can be fake and genuine supersymmetric. In the next subsection we construct some of the domain wall solutions as they will be related to the fractional brane solutions with non zero $H^{W}$.

\subsubsection{Some special domain wall flows}

A domain wall Ansatz is given by

$$
\mathrm{d} s_{D}^{2}=f^{2}(y) \mathrm{d} y^{2}+g(y)^{2} \eta_{\mu \nu} \mathrm{d} x^{\mu} \mathrm{d} x^{\nu},
$$

where $\eta$ is the metric on Minkowski space. We furthermore assume that the scalars only depend on $y$. Note that $f(y)$ is a gauge choice that corresponds to redefining the $y$ coordinate. For the presentation of the solution, we follow Bergshoeff et. al. [32] and choose the gauge $f=g^{2-p}$ to present the solutions. When we uplift to 10 dimensions below, we prefer to choice the conformal gauge $f=g$. To distinguish between both coordinates we use the coordinate $y$ in the Bergshoeff gauge and the coordinate $z$ in the conformal gauge.

In the language of [32] our effective action is a consistent truncation of an $\mathrm{SO}(2)$ gauging of maximal supergravity in 7 dimensions. The real effective theory of the smeared O6 compactification should however be a half-maximal supergravity in 7 dimensions. What counts here is that the bosonic fields of our truncation fit into the formalism of [32] where the solution is given in terms of two harmonic functions [32]

$$
h_{1}=2 \mathcal{H} y+\ell_{1}^{2}, \quad h_{2}=2 \mathcal{F} y+\ell_{2}^{2},
$$

as follows

$$
\begin{aligned}
g & =\left(h_{1} h_{2}\right)^{\frac{1}{4(p-1)}}, \\
\exp (x) & =\left(h_{1} h_{2}\right)^{\sqrt{\frac{p}{8(p-1)}}}, \\
\exp (u) & =\left(\frac{h_{1}}{h_{2}}\right)^{1 / 2} .
\end{aligned}
$$

By shifting the coordinate $y$ we can always set one of the two $\ell_{i}$ constants equal to zero.

A nice property of the solutions with both $\ell_{i}^{2}=0$, and $\mathcal{H}=\mathcal{F}$, is that they allow for a simple non-supersymmetric generalisation. This generalisation consists in deforming the supersymmetric solution (4.14), (4.15), (4.16) by rescaling the harmonic functions $h_{1}, h_{2}$ as follows

$$
h_{1,2} \rightarrow a h_{1,2} .
$$

To our knowledge these solutions are new. 
Let us now uplift the solutions to 10 dimensions. With the above reduction Ansatz this is straightforward. In what follows we restrict to $p=6$. As we anticipated, when the sources are smeared over the internal space $\left(H^{T}=0\right)$, the domain wall solution exactly gives the worldvolume dependence of the localised fractional brane solutions, after the coordinate redefinition

$$
y=\frac{\mathcal{F}}{2} z^{2} .
$$

and

$$
c=a \mathcal{F} .
$$

As we explained before the coordinate $z$ is the coordinate for which the domain wall metric is conformal to Minkowski $(f=g)$.

Hence we have reproduced the linear space-time dependence $H^{W}$ of the $10 \mathrm{D}$ warped solution using the smeared approximation. The linear dependence is only recovered for the solutions with both $\ell_{i}^{2}=0$ and $\mathcal{H}=\mathcal{F}$. These are the solutions with only one scalar, $x$, running and all other scalars fixed. More involved solutions will lift to other 10D solutions, which have not yet been constructed.

\subsubsection{Supersymmetry}

Note that the matching condition (4.19) is obeyed if one realises that our notation implied that for $p=6$ the flux density $\mathcal{F}$ is equal to the Romans mass $\mathcal{F}=m$. Then the supersymmetry of the domain wall solution corresponds to the supersymmetry condition for the JMO solution

$$
a=1 \quad \Longleftrightarrow \quad c=m
$$

\subsection{Interpretation}

The interpretation of dynamical fractional brane solutions as warped compactifications to $p+1$ dimensions allows a very simple understanding of:

1. Why the same linear $H^{W}$ is still possible when fluxes are added.

2. When supersymmetry requires the linear dependence in $H^{W}$.

Let us start with the first point. Consider dynamical $p$-brane solutions without fluxes. When interpreted as a warped compactification, ${ }^{5}$ the absence of fluxes implies the absence of a tree-level scalar potential; all scalars are free. Since the $x^{a}$-dependence for the fractional branes (the solution with flux) can be interpreted as the running of the lower-dimensional scalars, the same will be true for the solutions in which the scalars are free. What is then to be understood is why both the solutions with a scalar potential and the solutions without a scalar potential lift to the same 10-dimensional $H^{W}$ dependence. Since the $H^{W}$ dependence is purely generated by the lift of the scalar fields, it must be that the scalar fields have the same expression in both the solutions with flux and without flux. Indeed the domain wall solutions with both $\ell_{i}^{2}=0$ (4.14), (4.15), (4.16) and our non-supersymmetric

\footnotetext{
${ }^{5}$ For the rigorous reader who is worried about the tadpole condition one can think of placing some orientifold sources far away from the $\mathrm{D} p$ source.
} 
generalisation (4.17) are such that the scalars flow through the minimum of the scalar potential. More specifically, during the whole flow we have that

$$
V=0, \quad \partial_{u} V=0, \quad \partial_{x} V=0 .
$$

This means that on-shell there is no difference with the solutions of the free lower-dimensional theory. We leave the investigation of more general solutions that do not obey the above condition (4.21) for future work.

Similarly it is obvious to understand the solutions with the linear dependence in time (4.3). These are simply the cosmological solutions that obey (4.21). Since the domain/wall cosmology correspondence [33] flips the sign of $V$, but $V=0$ on shell, the same domain wall solutions can be Wick-rotated to cosmological solutions of the same supergravity theory. This then explains the linear $t$ behavior. For the case of $p=3$, which are warped compactifications to $D=4$, these are FLRW solutions that correspond to ultra-stiff cosmological fluids. In FLRW cosmological time $\tau$ these have the following scale factor

$$
a(\tau) \sim \tau^{\frac{1}{3}} .
$$

Second we discuss supersymmetry. Consider the JMO solution (3.14) for which we found a match between the original supersymmetry condition (3.15) discussed from a 10D point of view [24] and the supersymmetry of the corresponding domain wall solution in the reduced theory (4.20). Clearly, when the fluxes are zero then supersymmetry requires $H^{W}=0$. Hence it is truly the effect of the fluxes to introduce non-zero $H^{W}$ in order to satisfy the supersymmetry rules. Also this can be readily understood from the point of view of warped flux compactifications. Since the most well-known case is for compactifications down to $D=4$, we treat the case $p=3$ first.

As we discussed before, the interpretation of the lower-dimensional Minkowski vacuum is the 10D solution with $H^{W}=0$. Then it is well known that the ISD relation for the fluxes (3.2) is not sufficient for supersymmetry. There are extra constraints [1] on the fluxes and on the geometry: the internal geometry has to be conformal Calabi-Yau and the ISD fluxes have to point in a specific direction inside the Calabi-Yau space. In terms of the complex three-form $G=F_{3}-i e^{-\phi} H$, one requires $G$ to be of complexity type $(2,1)$ and primitive. A general ISD flux allows primitive $(2,1)$ directions and $(0,3)$-directions. Hence supersymmetry requires vanishing $(0,3)$ fluxes: ${ }^{6}$

$$
\star_{6} G_{3}=i G_{3}, \quad\left[G_{3}\right]_{0,3}=0 .
$$

This implies that for ISD fluxes that obey the above relation (4.23) we can have 10Dsolutions with $H^{W}=0$ that are supersymmetric. However, consider what happens when a non-vanishing $(0,3)$ piece is present. Then supersymmetry is consistent with the linear dependence in $H^{W}$. We can see this from the real superpotential $W$

$$
W=\left|\mathrm{e}^{\mathcal{K}} \mathcal{W}\right|, \quad \mathcal{W}=\int \Omega \wedge G,
$$

\footnotetext{
${ }^{6}$ The ISD condition here differs with a sign compared to earlier sections. Compare for example equation (3.6) of [20] (which agrees with the signs used in the following equation) with (3.2).
} 
with $\mathcal{K}$ the Kahler potential. A supersymmetric Minkowski solution satisfies

$$
\frac{\partial W}{\partial \phi^{i}}=0
$$

where the index $i$ runs over the scalar fields. When the flux has a $(0,3)$ piece this relation no longer can be satisfied since $W$ has no extremum. There is nonetheless another way to obtain supersymmetric solutions; one can allow the scalar fields to flow down the superpotential to create supersymmetric domain wall solutions. The supersymmetry condition then becomes

$$
\begin{aligned}
\dot{\phi}^{i} & =-f G^{i j} \frac{\partial W}{\partial \phi^{j}}, \\
\frac{\dot{g}}{g} & =\frac{1}{2(p-1)} f W .
\end{aligned}
$$

where a dot denotes a derivative with respect to the domain wall coordinate $y, G^{i j}$ is the inverse scalar field space metric and $f, g$ are the metric functions appearing in the domain wall metric (4.12). Therefore we find that, when a $(0,3)$ piece is present, one necessarily has to allow the scalars to flow in order to fulfill the supersymmetry conditions. This in turns implies the lower-dimensional metric is not Minkowski anymore but instead is of the domain wall type. The reason the 10-dimensional solution seems to have a $(p+1)$ dimensional Minkowski part (2.1) is simply because we used domain wall coordinates for which the metric is conformal to Minkowski $(f=g)$ and then the conformal part is absorbed in the warp factor (which indeed depends on all 10-dimensional coordinates).

Let us apply this to the specific choice of fluxes we made in the explicit example discussed in the previous section. The real superpotential is

$$
W=\exp \left(-\sqrt{\frac{p}{2(p-1)}} x\right)[\mathcal{H} \exp (-u)+\mathcal{F} \exp (+u)],
$$

and does not depend on $w \cdot{ }^{7}$ Indeed this superpotential does not allow a supersymmetric extremum since

$$
\partial_{x} W \neq 0
$$

as both $\mathcal{F}$ and $\mathcal{H}$ are positive. Instead its supersymmetric domain wall solutions correspond exactly to the solutions we gave previously, and which reproduced the linear dependence in $H^{W}$ of the JMO solution. The question remains as to what the uplift of the (supersymmetric)-domain wall solutions, for which $V$ and $\partial V$ are non-zero during the flow, correspond to. We leave this for future investigation.

\section{Outlook}

Let us conclude this paper by summarizing our results and what we can learn from it.

We have shown that the generalisation of extremal $p$-brane solutions to dynamical $p$ branes goes through in exactly the same way when fluxes are added to it that correspond

\footnotetext{
${ }^{7}$ This potential has the typical no-scale property $V=\frac{1}{2}\left(\partial_{u} W\right)^{2}$, where the massless fields at the no-scale Minkowski vacuum are $x$ and the decoupled field $w$. Only for $p=3$ this field corresponds to the volume modulus.
} 
to fractional $p$-branes. In practical terms this means that one can add a term linear in the worldvolume coordinates to the usual "harmonic function" $H$ that defines extremal solutions. ${ }^{8}$ Whenever the extra fluxes are present the possibility arises to trade the $\mathrm{D} p$-branes for $\mathrm{O} p$-planes and trading the non-compact transversal space for a compact one. This is a simple consequence of the RR tadpole condition that can be satisfied with $\mathrm{O} p$-planes and fractional brane type of fluxes. This establishes a map between the fractional brane type solutions in ten dimensions and warped compactifications. Exactly this map allowed us to interpret why the linear dependence of the worldvolume coordinates could be added to the harmonic function. This turns out to correspond to lower-dimensional solutions that are described by scalar fields that run along the massless direction in the minimum of the potential, as if the scalar is effectively free. This map furthermore provides a simple understanding of the supersymmetry conditions for this generalisation since it corresponds to the supersymmetry conditions in warped orientifold compactifications, which were known. ${ }^{9}$ Whenever the fluxes are such that the no-scale Minkowski vacuum is not supersymmetric then the solution must necessarily contain the worldvolume dependence in order to preserve supersymmetry. We have worked this out in detail for the simplest case of D6-branes (O6 planes) with $F_{0}$ and $H$ flux.

There are several interesting implications of our work. When our solutions are interpreted as warped compactifications they describe orientifold solutions with running moduli such that the orientifold backreaction has been taken into full account, whereas most solutions in the literature are vacuum solutions with constant scalar fields (see for instance $[20,30,36])$. Our solutions with running scalars are a natural $D$-dimensional extension of the existing solutions in four dimensions, most notably the solutions in $[5,11]$ and part of the solutions in [15]. The construction of such solutions is relevant for our understanding of flux compactifications since most flux compactifications are only understood in the limit where the sources are fully smeared. Such a limit takes into account the contribution of the tension to the four-dimensional energy and the contribution to the RR tadpoles, but nothing more. For supersymmetric or BPS-like no-scale vacua it has been noticed that the complex structure moduli are nonetheless unaltered by the full backreaction [20]. This is useful since it implies that at least the value of the cosmological constant and the position of the moduli can be trusted in the smeared limit. However this is probably all that can be trusted. Fluctuations around the vacuum, that for instance informs one about the moduli masses, cannot be trusted in the smeared limit and a warped effective field theory is therefore required. Furthermore one can expect that for non-supersymmetric (non-BPS like) solutions the moduli positions and possibly the vacuum energy do get altered when the backreaction is taken into account. ${ }^{10}$ Since the solutions in this paper feature running moduli one could wonder whether the running of the moduli is at all affected by the backreaction of the orientifold. For that purpose we have to compare the smeared solution

\footnotetext{
${ }^{8}$ The reason we put harmonic between quotation marks is that, in presence of fluxes, the Laplacian of $H$ is non-zero.

${ }^{9}$ This map between extremal $p$-brane solutions and (warped) flux compactifications fits is similar in spirit to the recently established link between black hole solutions and flux compactifications [34, 35].

${ }^{10}$ We refer to $[37,38]$ for some explicit investigations of backreaction for genuine non-BPS like solutions.
} 
from the localised one. Generically one does not expect the smeared effective action to contain information about the true warped effective action. However, we have found that the linear space-time dependence can be captured in the smeared approximation. This has a simple interpretation. The warped effective potential coincides with the KK-effective potential (the unwarped approximation) only at the minimum of both potentials. This is at least what concerns the scalar potentials of the lower-dimensional field theories. Besides the scalar potential one also expects changes in the kinetic terms for the scalars, due to warping (in fact the whole effective field theory should be different, even the degrees of freedom might differ [18]). It is not clear whether these 10-dimensional solutions might teach us anything about the kinetic terms of the lower-dimensional effective action.

It would be most interesting to construct solutions which are not linear in the spacetime coordinates. The interpretation of such solutions would be that they correspond to scalars flowing outside of the minimum of the scalar potential. It is likely that such solutions contain information about the scalar potential of the WEFT and the kinetic terms for the scalars, outside of the no-scale moduli space. We plan to report on this in a future publication.

\section{Acknowledgments}

We like to thank Iosif Bena, Mariana Graña, Hagen Triendl, Bret Underwood and Timm Wrase for useful discussions. JB is supported by the Göran Gustafsson Foundation. BJ is partially supported by the M.E.C. under contract FIS2010-17395 and by the Junta de Andalucía groups P07-FQM 03048 and FQM-6552. TVR and BV are supported respectively by the ERC Starting Independent Researcher Grant 259133-ObservableString and 240210-String-QCD-BH.

\section{A Ricci tensor}

Consider a metric Ansatz of the form

$$
\mathrm{d} s_{10}^{2}=e^{2 A(x, y)} \mathrm{d} s_{p+1}^{2}+e^{2 B(x, y)} \mathrm{d} s_{9-p}^{2}
$$

where $x$ is an external coordinate, and $y$ an internal. The Ricci tensor components for this metric are

$$
\begin{aligned}
R_{\mu \nu}= & \tilde{R}_{\mu \nu}-\mathrm{e}^{2(A-B)} \tilde{g}_{\mu \nu}\left(\left((p+1) \partial_{i} A+(7-p) \partial_{i} B\right) \tilde{g}^{i j} \partial_{j} A+\tilde{\nabla}_{y}^{2} A\right) \\
& -\tilde{g}_{\mu \nu}\left(\left((9-p) \partial_{\rho} B+(p-1) \partial_{\rho} A\right) \tilde{g}^{\rho \lambda} \partial_{\lambda} A+\tilde{\nabla}_{x}^{2} A\right) \\
& +\left(\left((9-p) \partial_{\mu} B+(p-1) \partial_{\mu} A\right) \partial_{\nu} A-(9-p)\left(\partial_{\mu} B-\partial_{\mu} A\right) \partial_{\nu} B\right) \\
& -\left((9-p) \nabla_{\mu} \partial_{\nu} B+(p-1) \nabla_{\mu} \partial_{\nu} A\right), \\
R_{\mu i}= & -p \partial_{\mu} \partial_{i} A-(8-p) \partial_{\mu} \partial_{i} B+8 \partial_{\mu} B \partial_{i} A,
\end{aligned}
$$




$$
\begin{aligned}
R_{i j}= & \tilde{R}_{i j}-\mathrm{e}^{2(B-A)} \tilde{g}_{i j}\left(\left((9-p) \partial_{\alpha} B+(p-1) \partial_{\alpha} A\right) \tilde{g}^{\alpha \beta} \partial_{\beta} B+\tilde{\nabla}_{x}^{2} B\right) \\
& -\tilde{g}_{i j}\left(\left((p+1) \partial_{k} B+(7-p) \partial_{k} B\right) \tilde{g}^{k l} \partial_{l} B+\tilde{\nabla}_{y}^{2} B\right) \\
& +\left(\left((p+1) \partial_{i} A+(7-p) \partial_{i} B\right) \partial_{j} B-(p+1)\left(\partial_{i} A-\partial_{i} B\right) \partial_{j} A\right) \\
& -\left((p+1) \nabla_{i} \partial_{j} A+(7-p) \nabla_{i} \partial_{j} B\right) .
\end{aligned}
$$

Both the metric and Ricci tensor are symmetric under the change

$$
\left.\begin{array}{c}
A(x, y) \\
p+1 \\
x^{\mu}
\end{array}\right\} \leftrightarrow\left\{\begin{array}{c}
B(x, y) \\
9-p \\
y^{i}
\end{array}\right.
$$

Open Access. This article is distributed under the terms of the Creative Commons Attribution License which permits any use, distribution and reproduction in any medium, provided the original author(s) and source are credited.

\section{References}

[1] S.B. Giddings, S. Kachru and J. Polchinski, Hierarchies from fluxes in string compactifications, Phys. Rev. D 66 (2002) 106006 [hep-th/0105097] [INSPIRE].

[2] S. Kachru, R. Kallosh, A.D. Linde and S.P. Trivedi, De Sitter vacua in string theory, Phys. Rev. D 68 (2003) 046005 [hep-th/0301240] [INSPIRE].

[3] O. DeWolfe and S.B. Giddings, Scales and hierarchies in warped compactifications and brane worlds, Phys. Rev. D 67 (2003) 066008 [hep-th/0208123] [INSPIRE].

[4] S.B. Giddings and A. Maharana, Dynamics of warped compactifications and the shape of the warped landscape, Phys. Rev. D 73 (2006) 126003 [hep-th/0507158] [INSPIRE].

[5] H. Kodama and K. Uzawa, Comments on the four-dimensional effective theory for warped compactification, JHEP 03 (2006) 053 [hep-th/0512104] [INSPIRE].

[6] K. Koyama, K. Koyama and F. Arroja, On the 4D effective theory in warped compactifications with fluxes and branes, Phys. Lett. B 641 (2006) 81 [hep-th/0607145] [INSPIRE].

[7] A.R. Frey and A. Maharana, Warped spectroscopy: Localization of frozen bulk modes, JHEP 08 (2006) 021 [hep-th/0603233] [INSPIRE].

[8] M.R. Douglas, J. Shelton and G. Torroba, Warping and supersymmetry breaking, arXiv:0704.4001 [INSPIRE].

[9] P. Koerber and L. Martucci, From ten to four and back again: How to generalize the geometry, JHEP 08 (2007) 059 [arXiv:0707.1038] [INSPIRE].

[10] M.R. Douglas and G. Torroba, Kinetic terms in warped compactifications, JHEP 05 (2009) 013 [arXiv: 0805.3700] [INSPIRE].

[11] F. Marchesano, P. McGuirk and G. Shiu, Open String Wavefunctions in Warped Compactifications, JHEP 04 (2009) 095 [arXiv: 0812.2247] [INSPIRE].

[12] F. Marchesano, P. McGuirk and G. Shiu, Chiral matter wavefunctions in warped compactifications, JHEP 05 (2011) 090 [arXiv:1012.2759] [INSPIRE]. 
[13] L. Martucci, On moduli and effective theory of $N=1$ warped flux compactifications, JHEP 05 (2009) 027 [arXiv:0902.4031] [INSPIRE].

[14] G. Shiu, G. Torroba, B. Underwood and M.R. Douglas, Dynamics of Warped Flux Compactifications, JHEP 06 (2008) 024 [arXiv:0803.3068] [INSPIRE].

[15] A.R. Frey, G. Torroba, B. Underwood and M.R. Douglas, The Universal Kähler Modulus in Warped Compactifications, JHEP 01 (2009) 036 [arXiv: 0810.5768] [inSPIRE].

[16] M.R. Douglas, Effective potential and warp factor dynamics, JHEP 03 (2010) 071 [arXiv:0911.3378] [INSPIRE].

[17] H.-Y. Chen, Y. Nakayama and G. Shiu, On D3-brane Dynamics at Strong Warping, Int. J. Mod. Phys. A 25 (2010) 2493 [arXiv:0905.4463] [InSPIRE].

[18] B. Underwood, A Breathing Mode for Warped Compactifications, Class. Quant. Grav. 28 (2011) 195013 [arXiv: 1009.4200] [INSPIRE].

[19] T.W. Grimm, D. Klevers and M. Poretschkin, Fluxes and Warping for Gauge Couplings in F-theory, arXiv: 1202.0285 [INSPIRE].

[20] J. Blaback, U.H. Danielsson, D. Junghans, T. Van Riet, T. Wrase and M. Zagermann, Smeared versus localised sources in flux compactifications, JHEP 12 (2010) 043 [arXiv: 1009.1877] [INSPIRE].

[21] M.R. Douglas and R. Kallosh, Compactification on negatively curved manifolds, JHEP 06 (2010) 004 [arXiv: 1001.4008] [INSPIRE].

[22] H. Kodama and K. Uzawa, Moduli instability in warped compactifications of the type IIB supergravity, JHEP 07 (2005) 061 [hep-th/0504193] [INSPIRE].

[23] P. Binetruy, M. Sasaki and K. Uzawa, Dynamical D4-D8 and D3-D7 branes in supergravity, Phys. Rev. D 80 (2009) 026001 [arXiv:0712.3615] [INSPIRE].

[24] B. Janssen, P. Meessen and T. Ortín, The D8-brane tied up: String and brane solutions in massive type IIA supergravity, Phys. Lett. B 453 (1999) 229 [hep-th/9901078] [INSPIRE].

[25] G. Gibbons, H. Lü and C. Pope, Brane worlds in collision, Phys. Rev. Lett. 94 (2005) 131602 [hep-th/0501117] [INSPIRE].

[26] I.R. Klebanov and A.A. Tseytlin, Gravity duals of supersymmetric $\mathrm{SU}(N) \times \mathrm{SU}(N+M)$ gauge theories, Nucl. Phys. B 578 (2000) 123 [hep-th/0002159] [INSPIRE].

[27] I.R. Klebanov and M.J. Strassler, Supergravity and a confining gauge theory: Duality cascades and chi SB resolution of naked singularities, JHEP 08 (2000) 052 [hep-th/0007191] [INSPIRE].

[28] M. Cvetič, G. Gibbons, H. Lü and C. Pope, Ricci flat metrics, harmonic forms and brane resolutions, Commun. Math. Phys. 232 (2003) 457 [hep-th/0012011] [INSPIRE].

[29] C.P. Herzog and I.R. Klebanov, Gravity duals of fractional branes in various dimensions, Phys. Rev. D 63 (2001) 126005 [hep-th/0101020] [INSPIRE].

[30] F. Saracco and A. Tomasiello, Localized O6-plane solutions with Romans mass, JHEP 07 (2012) 077 [arXiv: 1201.5378] [INSPIRE].

[31] R. D'Auria, S. Ferrara, F. Gargiulo, M. Trigiante and S. Vaula, $N=4$ supergravity Lagrangian for type IIB on $T^{6} / \mathbb{Z}_{2}$ in presence of fluxes and D3-branes, JHEP 06 (2003) 045 [hep-th/0303049] [INSPIRE]. 
[32] E. Bergshoeff, M. Nielsen and D. Roest, The Domain walls of gauged maximal supergravities and their M-theory origin, JHEP 07 (2004) 006 [hep-th/0404100] [INSPIRE].

[33] K. Skenderis and P.K. Townsend, Hidden supersymmetry of domain walls and cosmologies, Phys. Rev. Lett. 96 (2006) 191301 [hep-th/0602260] [INSPIRE].

[34] I. Bena, H. Triendl and B. Vercnocke, Camouflaged supersymmetry in solutions of extended supergravities, Phys. Rev. D 86 (2012) 061701 [arXiv:1111.2601] [INSPIRE].

[35] I. Bena, H. Triendl and B. Vercnocke, Black Holes and Fourfolds, JHEP 08 (2012) 124 [arXiv: 1206.2349] [INSPIRE].

[36] M. Graña, R. Minasian, M. Petrini and A. Tomasiello, A Scan for new $N=1$ vacua on twisted tori, JHEP 05 (2007) 031 [hep-th/0609124] [INSPIRE].

[37] J. Blaback, U.H. Danielsson, D. Junghans, T. Van Riet, T. Wrase and M. Zagermann, The problematic backreaction of SUSY-breaking branes, JHEP 08 (2011) 105 [arXiv:1105.4879] [INSPIRE].

[38] C. Burgess, A. Maharana, L. van Nierop, A. Nizami and F. Quevedo, On Brane Back-Reaction and de Sitter Solutions in Higher-Dimensional Supergravity, JHEP 04 (2012) 018 [arXiv: 1109.0532] [INSPIRE]. 